\title{
Controlling Systems with a Single Actuator
}

\author{
Alberto Portal and Pedro J. Zufiria
}

\begin{abstract}
In this paper the problem of designing a single actuator control for a class of systems is addressed. The existence of such control is studied and several ways for designing such control are provided. The results depend either on the rational canonical form or on the Jordan structure associated with the matrix which characterizes the system dynamics. The constructed control laws can be employed in the design of minimum cost controllers for a large variety of systems.
\end{abstract}

Keywords: Controllability $\bullet$ rational Frobenius form $\bullet$ Jordan form

\section{Introduction}

Dynamical system theory aims to characterize systems via time-evolution models $[1,2]$. Such characterization can be employed for performing two fundamental and dual tasks in dynamical system theory: on the one hand, the estimation of system's internal state from the measurement of accessible system outputs, defining the so called observation problem; on the other hand, the modification of system's internal state via the injection of appropriate system inputs, defining the so called control problem $[3,4,5]$. These problems have been thoroughly studied in the literature, specially for linear systems $[6,7,3]$. A fundamental issue when dealing with the control and observation of dynamical systems is that of the controllability and observability of the system, i.e. under which conditions a system can be controlled or observed. Such conditions are well established for the case of linear time invariant (LTI) dynamical systems of the form $\frac{d x}{d t}=A x(t)+B u(t), y(t)=C x(t)$, where $x(t) \in \mathbb{R}^{N}, u(t) \in \mathbb{R}^{R}$ and $y(t) \in \mathbb{R}^{L}$. Hence $B \in \mathbb{R}^{N \times R}$ and $C \in \mathbb{R}^{L \times N}$. The classical controllability condition for this system is stated as follows (see [3] for details): the system is controllable (i.e., the pair $(A, B)$ is controllable) if and only if the so called controllability matrix $\mathscr{C}(A, B):=\left(B|A B| A^{2} B|\cdots| A^{N-1} B\right) \in \mathbb{R}^{N \times(N R)}$, satisfies the condition $\operatorname{rank} \mathscr{C}(A, B)=N$. Many standard control problems have to deal with matrices $A$ and $B$ which are prestablished by the system dynamics and/or actuator restrictions. In this context, the controllability condition serves as a test on the existence of control laws with some specific properties. On the other hand, some applications or theoretical problems consider the possibility of arbitrarily selecting the number and location of the actuators. Hence, matrix $B$ may become a design element, and the controllability condition may serve as a design criterion. In 
the following section, we will study when matrix $B$ can only have a single column, meaning that a single controller can control the whole system.

\section{Systems Controllable with a Single Actuator}

Consider two matrices $A, B \in \mathbb{C}^{N \times N}$, and the associated controllability matrix $\mathscr{C}(A, B) \in$ $\mathbb{C}^{N \times N^{2}}$. Suppose that $A$ is a fixed given matrix (e.g., determined by the structure of a LTI dynamical system), so that we can simplify the notation by writing $\mathscr{C}(B)$ instead of $\mathscr{C}(A, B)$. We want to determine when there exists a rank one matrix $B$ so that $\operatorname{rank}[\mathscr{C}(B)]=N$, and also to construct a matrix $B$ satisfying such condition. This is equivalent to finding a non-zero vector $b \in \mathbb{C}^{N}$ such that $\operatorname{rank}[\mathscr{C}(b)]=N$. In such a case, the system could be controlled with a single actuator.

\subsection{The Rational Canonical Form Perspective}

Let $p_{A}(\lambda)=c_{0}+c_{1} \lambda+\cdots+c_{N-1} \lambda^{N-1}+\lambda^{N}$ be the characteristic polynomial associated with matrix $A$. In general, matrix $A$ may or may not be similar to the corresponding companion matrix $C\left(p_{A}\right)$ form of its characteristic polynomial [3]. In any case, it is always similar to a block diagonal matrix whose blocks take the companion form. Such a matrix is the rational canonical form (also called Frobenius form); this rational canonical form provides a minimal decomposition of the vector space into cyclic subspaces, each one of them spanned by some vector and its repeated images under $A$. This decomposition is very useful in characterizing controllability of pairs $(A, B)$. Note that the rational canonical form does not require to work with an algebraically closed field (allowing a characteristic polynomial factorization). We now address the single actuator problem.

Let us denote $e_{i}[N]$ the $i$-th column of the identity matrix, $I_{N}$, or simply $e_{i}$ if there is no ambiguity. Note that if $A$ is a companion matrix, then if we choose $b=e_{1}[N]$ we obtain $\mathscr{C}\left(A, e_{1}[N]\right)=I_{N}$ (full rank) so that $(A, b)$ is controllable. More generally, if $b=w$ with $w_{1} \neq 0$ and $w_{N}=0$, it generates a full-rank lower triangular matrix $\mathscr{C}(A, B)$, making the pair $(A, b)$ also controllable. In general, it is is known that the following statements are equivalent: a) $A$ is similar to the companion matrix of its characteristic polynomial; b) the characteristic polynomial coincides with the minimal polynomial of $A$ (i.e., the minimal polynomial has degree $N$ ); c) there exist a cyclic vector $v$ such that $\left\{v, A v, A^{2} v, \ldots, A^{n-1} v\right\}$ is a basis of $\mathbb{R}^{N}$. Note that if there exists a vector $v$ such that $\left\{v, A v, A^{2} v, \ldots, A^{n-1} v\right\}$ is a basis of $\mathbb{R}^{N}$, then $v$ satisfies the condition $\operatorname{rank} \mathscr{C}(A, v)=N$. Based on this result we can state the following:

Proposition 1 Matrix $A$ can be controlled with a single controller iff it is similar to the companion matrix of its characteristic polynomial.

Hence, in order to determine the one-actuator controllability of the system defined by matrix $A$, it suffices to check if $A$ is similar to the companion matrix of its characteristic polynomial. The computation of a cyclic vector $v($ or $b)$ which satisfies rank $\mathscr{C}(A, v)=N$ can be carried out by considering the similarity transformation $C\left(p_{A}\right)=T^{-1} A T$ where $T$ is a regular transformation matrix. Note that defining $x=T z$, the equation of the system takes the form $\frac{d z}{d t}=T^{-1} A T x(t)+T^{-1} B u(t)=$ 
$C\left(p_{A}\right) z(t)+T^{-1} B u(t)$. Hence, since, as mentioned earlier, the pair $\left(C\left(p_{A}\right), w\right)$ (with $w_{1} \neq 0$ and $\left.w_{N}=0\right)$ is controllable, this design procedure provides $B=b=T w$ as a set of solutions for the single actuator problem. In the following, we address the controllability analysis by resorting to the alternative Jordan canonical form.

\subsection{Alternative Approach. The Jordan Form}

The Jordan canonical form requires to work with an algebraically closed field, such as the set of complex numbers. Nevertheless, this may not be a critical aspect in many applications, and the Jordan form is a more well known canonical form reference for similarity analysis between matrices. In the following, we illustrate its use in controllability analysis. Since $A$ can be written as $P J P^{-1}$ where $J$ stands for the Jordan canonical form of $A$, it is clear that $\mathscr{C}(B)$ and $P^{-1} \mathscr{C}(B)$ have the same rank and the pair $B$ and $P^{-1} B$ have the same number of columns; hence, it is sufficient to study the case where $A$ is in Jordan canonical form. We begin by introducing some basic notation: $\mathfrak{B}_{n}^{m}(\lambda):=\left(\begin{array}{c}m \\ n\end{array}\right) \lambda^{m-n}$, if $0 \leq n \leq m$ and $\mathfrak{B}_{n}^{m}(\lambda):=0$, otherwise, for $m, n \in \mathbb{Z}$ and $\lambda \in \mathbb{C}$, where $\left(\begin{array}{l}m \\ n\end{array}\right)$ stands for the binomial coefficient $\frac{m !}{n !(m-n) !}$. As usual, we also denote the Jordan block of size $N \in \mathbb{N}$ and diagonal entry $\lambda \in \mathbb{C}$ as $J_{\lambda, N}$. Denote $Q_{N}:=J_{0, N}$ and define $Q_{N}^{0}:=I_{N}$, i.e. the identity matrix of order $N$. It is well known that $\left(Q_{N}^{M}\right)_{i, j}$, i.e., the element of $Q_{N}^{M}$ in the $i$-th row and the $j$-th column, is 1 if $i-j=M$ and 0 otherwise. Notice that, for $M \geq N, Q_{N}^{M}=O_{N}$, the zero matrix of order $N$. Then, if we write $J_{\lambda, N}=\lambda Q_{N}^{0}+Q_{N}$, it is straightforward to compute its powers $J_{\lambda, N}^{M}=\left(\lambda Q_{N}^{0}+Q_{N}\right)^{M}=\sum_{m=0}^{M}\left(\begin{array}{c}M \\ m\end{array}\right) \lambda^{M-m} Q_{N}^{m}=\sum_{m=0}^{M} \mathfrak{B}_{m}^{M}(\lambda) Q_{N}^{m}$, which reduces to $\sum_{m=0}^{N-1} \mathfrak{B}_{m}^{M}(\lambda) Q_{N}^{m}$ when $M \geq N$. As defined above, $e_{i}[N]$ or $e_{i}$ will stand for the $i$-th column of the identity matrix, $I_{N}$. We are now ready for demonstrating the conditions that the Jordan canonical form of matrix $A$ must satisfy in order to guarantee the existence of a single actuator control. In addition, a design method for such existing single actuator is also provided.

In the next lemma we determine the rank of $\mathscr{C}\left(J_{\lambda, N}, e_{1}[N]\right)$ by showing its triangular structure. As a more general controllability-like matrix will be needed, we establish the following result in terms of such an extended controllability matrix. Concretely, for $A \in \mathbb{C}^{N \times N}, B \in \mathbb{C}^{N \times R}$ and $M \geq N$, we define $\mathscr{C}_{M}(A, B):=$ $\left(B, A B, A^{2} B, A^{3} B, \cdots, A^{N-1} B, \cdots, A^{M-1} B\right) \in \mathbb{C}^{N \times M R}$.

Lemma 1 Consider $M \geq N$ and $\lambda \in \mathbb{C}$. The element of the matrix $\mathscr{C}_{M}\left(J_{\lambda, N}, e_{1}\right)$ in the $i$-th row and the $j$-th column, $\left[\mathscr{C}_{M}\left(J_{\lambda, N}, e_{1}\right)\right]_{(i, j)}$, is given by $\left(\begin{array}{c}j-1 \\ i-1\end{array}\right) \lambda^{j-i}$ when $i \leq j$ and 0 otherwise. Moreover, $\operatorname{rank}\left[\mathscr{C}_{M}\left(J_{\lambda, N}, e_{1}\right)\right]=N$.

Proof. This result is obvious from the expression for the powers since $J_{\lambda, N}^{m} e_{1}$ is the first column of the matrix $J_{\lambda, N}^{m}$. Notice that the $i$-th row of $\mathscr{C}_{M}\left(J_{\lambda, N_{,},} e_{1}\right)$ is given by $\left[\mathscr{C}_{M}\left(J_{\lambda, N}, e_{1}\right)\right]_{(i,)}=\left(\mathfrak{B}_{i-1}^{0}(\lambda) \cdots \mathfrak{B}_{i-1}^{i-1}(\lambda) \mathfrak{B}_{i-1}^{i}(\lambda) \mathfrak{B}_{i-1}^{i+1}(\lambda) \cdots \mathfrak{B}_{i-1}^{M-1}(\lambda)\right)$, so $\mathscr{C}_{M}\left(J_{\lambda, N}, e_{1}\right)$ has an upper triangular structure with ones in its diagonal, and its rank is $N$.

We now prove that in fact $e_{1}$ can be replaced by any $v \in \mathbb{C}^{N}$ such that $v_{1} \neq 0$ in Lemma 1 and the conclusion remains true. 
Lemma 2 Consider $L \in \mathbb{N}, v \in \mathbb{C}^{L}, \lambda \in \mathbb{C}$ and the matrix $\mathscr{C}_{M}\left(J_{\lambda, L}, v\right)$, with $M \geq$ $L-1$. Then, for $i=1,2, \ldots, L$, the $i$-th row of $\mathscr{C}_{M}\left(J_{\lambda, L}, v\right)$ is a linear combination of the first $i$ rows of the matrix $\mathscr{C}_{M}\left(J_{\lambda, L}, e_{1}\right)$. Precisely, for $1 \leq i \leq L$, we have $\left[\mathscr{C}_{M}\left(J_{\lambda, L}, v\right)\right]_{(i,:)}=\sum_{\ell=1}^{i} v_{i-\ell+1}\left[\mathscr{C}_{M}\left(J_{\lambda, L}, e_{1}\right)\right]_{(\ell,:)}$.

Proof. Consider $v=\left(v_{1}, v_{2}, \ldots, v_{L}\right)^{\top} \in \mathbb{C}^{L}$. We obtain that, if $1 \leq j \leq M+1$,

$$
J_{\lambda, L}^{j-1} v=\left[\sum_{k=0}^{j-1}\left(\begin{array}{c}
j-1 \\
k
\end{array}\right) \lambda^{j-1-k} Q_{L}^{k}\right]\left(\sum_{\ell=1}^{L} v_{\ell} e_{\ell}\right)=\sum_{\ell=1}^{L} v_{\ell}\left[\sum_{k=0}^{j-1}\left(\begin{array}{c}
j-1 \\
k
\end{array}\right) \lambda^{j-1-k}\right] Q_{L}^{k} e_{\ell} .
$$

Taking into account that $\left[Q_{L}^{\ell}\right]_{(i, j)}$ is 1 when $i-j=\ell$ and 0 otherwise, the $i$-th element of the $j$-th column of $\mathscr{C}_{M}\left(J_{\lambda, L}, v\right)$, where $1 \leq i \leq L$ and $1 \leq j \leq M$, is given by: $\left[J_{\lambda, L}^{j-1} v\right]_{(i)}=e_{i}^{\top} J_{\lambda, L}^{j-1} v \sum_{\ell=\max \{1, i-j+1\}}^{i} v_{\ell}\left(_{i-\ell}^{j-1}\right) \lambda^{j-1-i+\ell}=\sum_{\ell=1}^{i} v_{\ell} \mathfrak{B}_{i-\ell}^{j-1}(\lambda)$. Then we have that $\left[\mathscr{C}_{M}\left(J_{\lambda, L}, v\right)\right]_{(i,:)}=\sum_{\ell=1}^{i} v_{i-\ell+1}\left[\mathscr{C}_{M}\left(J_{\lambda, N}, e_{1}\right)\right]_{(\ell,:)}$, which concludes the proof.

The next theorem solves our problem when we have exactly one Jordan block for each eigenvalue of $A$. In this case, we have $R=1$.

Theorem 1 Let $\lambda_{1}, \lambda_{2}, \ldots, \lambda_{K}$ be $K$ different complex numbers and $N_{1}, N_{2}, \ldots, N_{K} \in$ $\mathbb{N}$. Consider the matrix $J=J_{\lambda_{1}, N_{1}} \oplus J_{\lambda_{2}, N_{2}} \oplus \cdots \oplus J_{\lambda_{K}, N_{K}}$. Define e $:=$ $\left(e_{1}\left[N_{1}\right]^{\top} e_{1}\left[N_{2}\right]^{\top} \cdots e_{1}\left[N_{K}\right]^{\top}\right)^{\top}$ and $N:=\sum_{k=1}^{K} N_{k}$. Then, the matrix $\mathscr{C}(J, \mathbf{e})$ has $\operatorname{rank} N$.

Proof. First, notice that if we denote $f_{n}(z):=z^{n}$ for $z \in \mathbb{C}$ and $n \in \mathbb{N} \cup\{0\}$, then, for $1 \leq k \leq K,\left[\mathscr{C}\left(J_{\lambda_{k}, N_{k}}, e_{1}\left[N_{k}\right]\right)\right]_{(1,:)}=\left(f_{0}\left(\lambda_{k}\right) f_{1}\left(\lambda_{k}\right) \cdots f_{N-1}\left(\lambda_{k}\right)\right)$ and $\left[\mathscr{E}\left(J_{\lambda_{k}, N_{k}}, e_{1}\left[N_{k}\right)\right]_{(i,)}\right.$ $=\left(\frac{1}{(i-1) !} f_{0}^{(i-1)}\left(\lambda_{k}\right) \frac{1}{(i-1) !} f_{1}^{(i-1)}\left(\lambda_{k}\right) \cdots \frac{1}{(i-1) !} f_{N-1}^{(i-1)}\left(\lambda_{k}\right)\right)$ for $i=2,3, \ldots, N_{k}$. Write $0 \in \mathbb{C}^{N}$ as a linear combination of the columns of $\mathscr{C}(J, \mathbf{e}): \sum_{n=1}^{N} \alpha_{n}[\mathscr{C}(J, \mathbf{e})]_{(:, n)}=0$, where $\alpha_{1}, \alpha_{2}, \ldots$, $\alpha_{N} \in \mathbb{C}$. ¿From the above equations we deduce that, for each $k \in\{1,2, \ldots, K\}$, the eigenvalue $\lambda_{k}$ is a root of the polynomial $p(z):=\sum_{n=1}^{N} \alpha_{n} z^{n-1}$ whose multiplicity is $N_{k}$, so $p(z)$ must be the zero polynomial as its degree is $N-1$ and, since the $K$ eigenvalues are different each other, it has $N$ roots.

Corollary 1 Let $A \in \mathbb{C}^{N \times N}$ has $K$ different eigenvalues $\lambda_{1}, \lambda_{2}, \ldots, \lambda_{K} \in \mathbb{C}$ and suppose that its Jordan canonical form is $J=J_{\lambda_{1}, N_{1}} \oplus J_{\lambda_{2}, N_{2}} \oplus \cdots \oplus J_{\lambda_{K}, N_{K}}$, where $\sum_{k=1}^{K} N_{k}=N$. Then, the minimum rank of matrix $B$ for the controllability matrix $\mathscr{C}(A, B)$ to have rank $N$ is $R=1$.

Again, a vector $b$ which satisfies $\operatorname{rank} \mathscr{C}(A, b)=N$ can be computed via the similarity transformation $J=T^{-1} A T^{\prime}$ where $T^{\prime}$ is a regular transformation matrix. Defining $x=T^{\prime} w$, the equation of the system becomes $\frac{d w}{d t}=T^{\prime-1} A T^{\prime} w(t)+$ $T^{\prime-1} B u(t)=J w(t)+T^{\prime-1} B u(t)$. Now, considering Lemma 2 and Theorem 1, the pair $\left(C\left(p_{A}\right), \mathbf{v}\right)$ with $\mathbf{v}:=\left(v_{1}^{\top} v_{2}^{\top} \cdots v_{K}^{\top}\right)^{\top}$ so that $v_{k} \in \mathscr{R}^{N_{k}}, v_{k, 1} \neq 0$ and $N:=$ 
$\sum_{k=1}^{K} N_{k}$, is controllable, and $B=b=T^{\prime} \mathbf{v}$ provides also an alternative computation for a set of solutions for the single actuator problem.

\subsection{Relationship between Both Approaches}

The rational and the Jordan canonical forms approaches lead to equivalent results concerning the conditions of existence of solution. The condition of matrix $A$ having a single Jordan block associated with each eigenvalue is equivalent to the condition of the characteristic polynomial being also the minimal polynomial; hence, when Theorem 1 applies, so does the equivalence of statements concerning the companion matrix. Both procedures provide different solutions for the computation of a matrix $b$ satisfying the controllability condition.

\section{Concluding Remarks}

We have studied the problem of designing a single actuator control for a class of dynamical systems. The existence of such control has been studied and several ways for designing it have been provided. The results are grounded on the rational canonical form or on the Jordan structure associated with the matrix which characterizes the system dynamics. The obtained control laws can be employed in the design of minimum cost controllers for a large variety of systems.

Acknowledgments. The authors want to acknowledge the financial support of Orange (Spain and France), via the Cátedra Orange at the ETSIT-UPM, Spain, and the support via project MTM2010-15102 of MCeI, Spain.

\section{References}

1. Luenberger, D. (1979) Introduction to dynamic systems: theory, models, and applications. Wiley

2. Vidyasagar, M. (2002) Nonlinear systems analysis. SIAM Vol. 42

3. Friedland, B. (1986) Control System Design (1986) An introduction to state space methods. McGraw-Hill, Inc

4. Liu, Yang-Yu, Slotine, J.J. and Barabási, A.-L. (2011) Controllability of complex networks. Nature 473(7346):167-173

5. Weber, Thomas A. (2011) Optimal Control Theory with Applications in Economics, MIT Press

6. Kalman, R. E. (1963) Mathematical description of linear dynamical systems. Journal of the SIAM, Series A: Control, 1(2):152-192

7. Chen, C.-T. (1995) Linear system theory and design. Oxford University Press, Inc 